\title{
Experiencia clínica en tiroidectomía total del Servicio de Otorrinolaringología y Cirugía de Cabeza y Cuello Hospital Guillermo Grant Benavente
}

\section{Clinical experience in total thyroidectomy of the Department of Otolaryngology and Head and Neck Surgery of Guillermo Grant Benavente Hospital}

\author{
Gabriel Undurraga $\mathbf{P}^{1}$, Felipe Fredes $\mathbf{C}^{1}$, Pablo Ortega $\mathbf{R}^{1}$, Alexis Urra $\mathbf{B}^{1}$, Álvaro Compan $\mathrm{J}^{2}$, \\ Joaquín Ulloa $S^{3}$, Ignacio Mendoza A $^{1}$.
}

\begin{abstract}
RESUMEN
Introducción: La tiroidectomía es una de las cirugías más frecuentes realizadas en cabeza y cuello. Existen escasos reportes de experiencia en tiroidectomías en servicios de otorrinolaringología en Chile.

Objetivo: Analizar la experiencia clínica con las tiroidectomías totales realizadas en el Servicio de ORL y Cirugía de Cabeza y Cuello de nuestro hospital.

Material y método: Estudio descriptivo retrospectivo. Revisión de fichas clínicas de pacientes sometidos a tiroidectomías totales (TT) entre los años 2010 y 2014 en nuestro servicio.

Resultados: Se realizaron 271 tiroidectomías totales. La indicación más frecuente fue por tamaño (51\%). Los diagnósticos más frecuentes fueron carcinomas papilares (46\%) e hiperplasia folicular coloidea (37\%). Se presentaron lesiones transitorias del nervio laríngeo recurrente en 2,9\%, y lesiones persistentes en 2,5\%. Hipocalcemia transitoria se presentó en el $25 \%$ de los pacientes.

Discusión: Nuestra serie presenta resultados concordantes con la literatura, presenta una baja tasa de complicaciones, las cuales se observan principalmente en pacientes con vaciamiento cervical y/o en que se observaron 2 o menos paratiroides.

Conclusiones: La tiroidectomía total es una técnica quirúrgica segura y nuestra experiencia presenta resultados concordantes a los publicados en la literatura internacional.
\end{abstract}

Palabras clave: Tiroidectomía total, hipocalcemia, lesión nervio laríngeo recurrente.

1 Medico Servicio Otorrinolaringólogia, Hospital Guillermo Grant Benavente.

2 Médico Cirugía de Cabeza y Cuello, Hospital Guillermo Grant Benavente.

3 Médico, Hospital Guillermo Grant Benavente.

Recibido el 23 de marzo de 2016. Aceptado el 22 de junio de 2016. 


\section{ABSTRACT}

Introduction: Thyroidectomy is one of the most common surgeries performed in head and neck. There are few reports of experience in thyroidectomy in otolaryngology services in Chile.

Aim: To analyze the clinical experience with total thyroidectomy performed in the service of ENT and Head and Neck Surgery of our hospital.

Material and method: Retrospective descriptive study. Review of medical records of patients undergoing total thyroidectomy (TT) between 2010 and 2014 in our service records.

Results: A total of 271 thyroidectomy were performed. The most frequent indication was size (51\%). The most frequent diagnoses were papillary carcinomas (46\%) and follicular colloid hyperplasia (37\%). We found $2.5 \%$ of transient recurrent laryngeal nerve injury and $2.9 \%$ had persistent lesions. Transient hypocalcemia occurred in $25 \%$ of patients.

Discussion: Our series shows good agreement with literature, it has a low rate of complications, which are mainly seen in patients with neck dissection and / or observed 2 or less parathyroid during surgery.

Conclusions: Total thyroidectomy is a safe surgical technique, and our experience shows consistent results to those reported in international literature.

Key words: Total thyroidectomy, hypocalcemia, recurrent laryngeal nerve injury.

\section{INTRODUCCIÓN}

La tiroidectomía es una de las cirugías más frecuentes realizadas en cirugía de cabeza y cuello. En muchos centros alrededor del mundo es realizada por otorrinolaringólogos. Sin embargo, existen escasos reportes de experiencia en tiroidectomías en servicios de otorrinolaringología en Chile, siendo la serie más grande en nuestro país la publicada en 2011 por Ortega y cols del Servicio de Otorrinolaringología y Cirugía de Cabeza y Cuello del Hospital Guillermo Grant Benavente'1.

Si es realizada por equipos especializados es una cirugía segura con baja tasa de morbilidad y prácticamente cero mortalidad².

La tiroidectomía puede ser total o parcial. La total cada vez es más aceptada como la técnica de elección tanto para patología benigna como maligna ${ }^{3}$.

Las indicaciones más frecuentes incluyen: Cáncer de tiroides, bocio multinodular y enfermedad de Graves.

Las complicaciones son directamente proporcionales a la extensión de la cirugía e inversamente proporcional a la experiencia del cirujano².

Las complicaciones se dividen en transitorias y permanentes según si permanecen por más de
6 meses o no. Las más frecuentes son: hipoparatiroidismo transitorio $(7 \%-40 \%)$ y permanente $(0 \%$ $5 \%)^{4}$; lesión de nervio laríngeo recurrente (NLR) transitoria $(5 \%-7 \%)$ y permanente $(0 \%-2 \%)^{5}$; además la ocurrencia de hematomas cervicales $(0,3 \%$ $3 \%)^{6}$. Si se asocia la tiroidectomía a un vaciamiento cervical, la tasa de complicaciones aumenta ${ }^{7}$.

\section{OBJETIVO}

Nuestro objetivo general es analizar la experiencia clínica en las tiroidectomías totales realizadas en el Servicio de Otorrinolaringología y Cirugía de Cabeza y Cuello de nuestro hospital, centrándonos principalmente en conocer los antecedentes clínicos (diagnóstico de derivación, estudio preoperatorio e indicación quirúrgica), caracterizar la técnica quirúrgica y los hallazgos intraoperatorios, y finalmente determinar la tasa de complicaciones.

\section{MATERIAL Y MÉTODO}

Estudio descriptivo retrospectivo. Se incluyeron a todos los pacientes sometidos a tiroidectomía entre el 
1 enero de 2010 hasta el 31 de diciembre de 2014 en el Servicio de Otorrinolaringología y Cirugía de Cabeza y Cuello del Hospital Guillermo Grant Benavente. Se excluyeron las resecciones tiroideas parciales $y / 0$ subtotales. Se revisó la ficha clínica de cada paciente, registrando datos epidemiológicos. Se recopiló información sobre el diagnóstico de derivación, estudio preoperatorio, indicación quirúrgica, técnica quirúrgica, hallazgos intraoperatorios, evolución posoperatoria, y resultado final de la pieza operatoria. Como norma de nuestro servicio, se realizó estudio de calcemia posoperatoria cada 12 horas hasta el alta clínica, acompañado de un estudio endoscópico de la motilidad cordal en los casos que presentaron disfonía posoperatoria. Se definió como lesión del NLR a toda alteración de la motilidad cordal que apareciera en el posoperatorio. Se definió como hipocalcemia a un calcio menor a $8 \mathrm{mg} / \mathrm{dl}$. En cuanto al seguimiento ambulatorio, el primer control se realizó a las 3 semanas con el resultado de la biopsia asociado a la calcemia del paciente. Se definió a las complicaciones como transitorias si remitían dentro de los primeros 6 meses posoperatorios y como persistentes si permanecían más allá de ese período. Todos los datos fueron registrados en una tabla Excel. Se realizó un análisis estadístico con software SPSS v22. Se consideró estadísticamente significativo $\mathrm{p}<0,05$.

\section{RESULTADOS}

Se realizaron 300 tiroidectomías en nuestro servicio, de las cuales se excluyeron 29 casos por ser resecciones tiroideas parciales y/o subtotales, dando un total de 271 tiroidectomías totales estudiadas.
El rango de edad fue de 15 a 89 años, con un promedio de edad de 48,6 años. El $89 \%$ de los pacientes eran de sexo femenino.

El $100 \%$ de los pacientes se le realizó estudio con ecotomografía tiroidea. El $56 \%$ de los pacientes presentó al menos un nódulo tiroideo mayor a 3 centímetros de diámetro.

En el $91 \%$ de los casos se realizó punción aspirativa con aguja fina (PAAF) cuyos resultados se informaron según la clasificación de Bethesda. Los con mayor incidencia fueron los grupos Bethesda $2(32,8 \%)$ y Bethesda $6(19,4 \%)$.

La indicaciones quirúrgicas fueron: tamaño aumentado (nódulo tiroideo mayor a 3 centímetros) en $58 \%$; alta sospecha de cáncer (PAAF con Bethesda 4,5 ó 6 , asociado a características ecográficas de malignidad) en el 38\%; cáncer confirmado (biopsia previa compatible con carcinoma tiroideo) en el $3 \%$; y oftalmopatía distiroidea en solo $1 \%$.

La técnica quirúrgica utilizada en todos los casos fue la siguiente: una incisión cervical tipo Kocher, luego sección de músculos pretiroideos, disección subcapsular de glándula tiroides, ligadura de pedículos vasculares previo reconocimiento de NLR y de glándulas paratiroides, hemostasia con ligaduras, bipolar y Harmonic scalpel Focus ${ }^{\circledR}$, y finalmente dren aspirativo subcutáneo. En solo el $12 \%$ de los casos se asoció un vaciamiento cervical. En esos casos, se amplió la incisión, luego una disección ganglionar de los niveles comprometidos, dejando 2 drenajes aspirativos en los casos de vaciamientos cervicales bilaterales (66\%).

En la Figura 1 se desglosa la cantidad de cirugías realizadas por año, siendo el 2012 el año con mayor número de intervenciones realizadas.

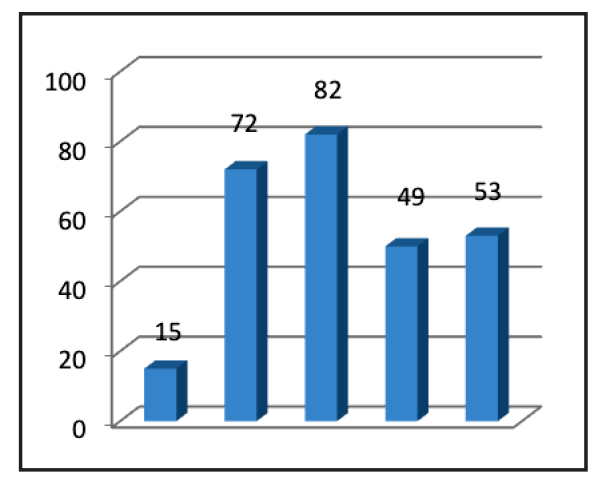

Figura 1. Número de cirugías por año. 
En el $94 \%$ de los casos se observó 3 o más paratiroides durante la cirugía.

En el 9,2\% de los casos se observó extensión tumoral extratiroidea, siendo la estructura más comprometida el NLR en 12 casos, seguido de la tráquea en 8 casos y el esófago en 5 casos.

El tiempo quirúrgico promedio fue de 2 horas y 15 minutos, el cual se prolongó en promedio a 5 horas en los casos asociados a vaciamiento cervical.

En cuanto a la evolución clínica, la duración del posoperatorio hospitalizado fue en promedio de 2,9 días, y se prolongó a 5,9 días en promedio en los casos que se asoció a un vaciamiento cervical. La cantidad de días con drenaje fue de 2,2 y 3,7 días, respectivamente.

Respecto a las complicaciones, hubo solo una complicación intraoperatoria $(0,36 \%)$ que consistió en una lesión esofágica, la cual se reparó con sutura primaria. En el $26 \%$ del total de pacientes se presentó una complicación transitoria (Figura 2) siendo la más frecuente la hipocalcemia, las cuales fueron asintomáticas, se presentaron en las primeras 48 horas, prolongando la estadía posoperatoria a 4,8 días en promedio, y todos fueron dados de alta con calcemias dentro del rango de

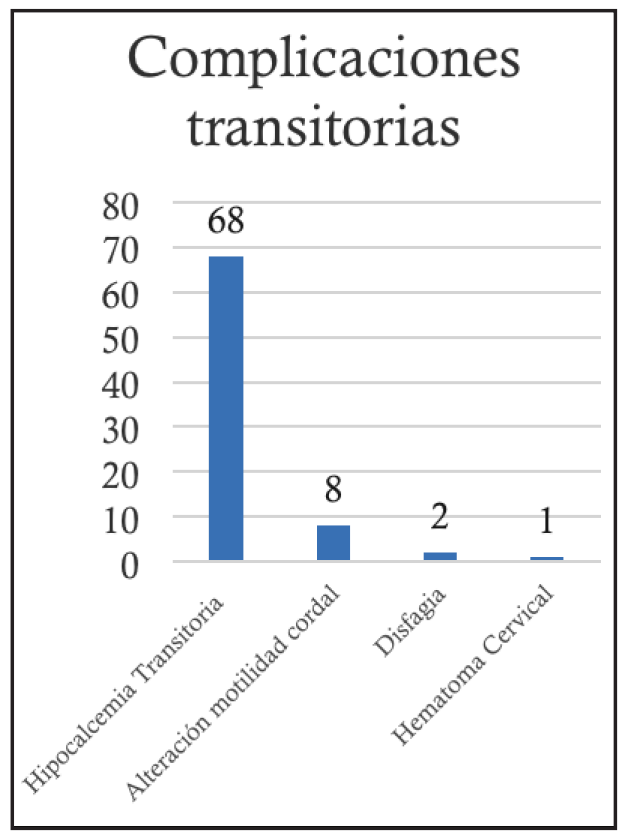

Figura 2. Complicaciones transitorias. la normalidad. En menor frecuencia lesión de NLR, disfagia y hematoma cervical. Las complicaciones persistentes se presentaron en el $5,5 \%$ de los casos, siendo la más frecuente la lesión de NLR, seguida por la cicatriz patológica e hipoparatiroidismo posquirúrgico (Figura 3).

Los diagnósticos histopatológicos más frecuentes fueron carcinoma papilar (46\%) e hiperplasia folicular coloidea (37\%) como se detalla en Tabla 1.

Las complicaciones se presentaron en mayor frecuencia en aquellos pacientes en que se realizó un vaciamiento cervical $(p 0,002) \mathrm{y} / 0$ se observaron 2 o menos paratiroides ( $p 0,018)$. La edad y el sexo no influyeron en la incidencia de cáncer en nuestra serie $(p>0,05)$.

\section{DISCUSIÓN}

Nuestra experiencia clínica en tiroidectomía total presenta resultados concordantes con los de la literatura mundial. Esta serie es la más grande

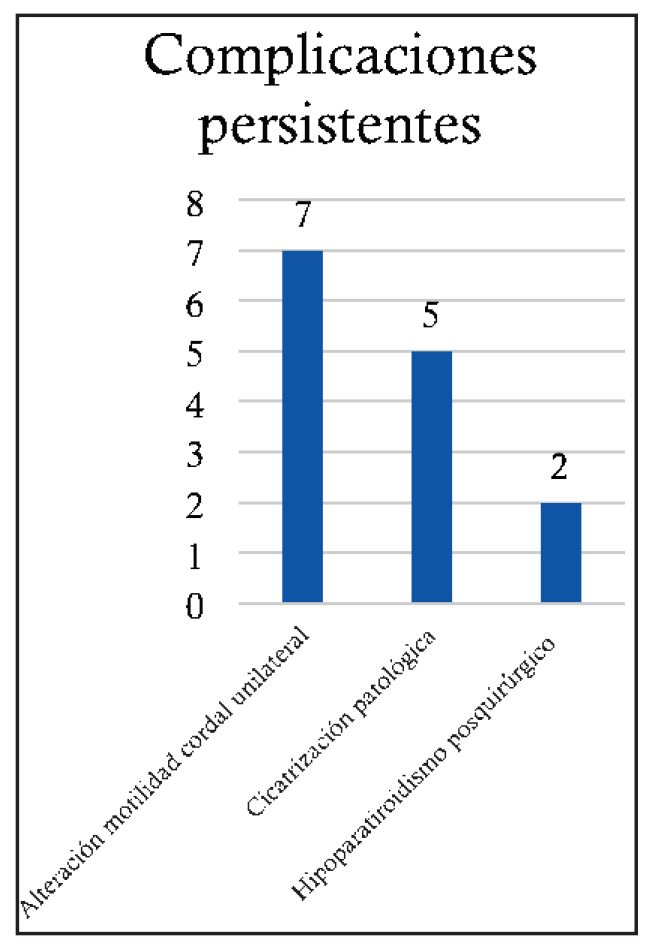

Figura 3. Complicaciones persistentes. 
Tabla 1. Diagnóstico histopatológico

\begin{tabular}{|lrc|}
\hline Diagnóstico histopatológico & N & $\%$ \\
\hline Carcinoma papilar & 125 & 46 \\
Hiperplasia folicular coloidea & 101 & 37 \\
Adenoma folicular & 23 & 9 \\
Tiroiditis de Hashimoto & 8 & 3 \\
Carcinoma folicular & 7 & 2,4 \\
Carcinoma medular & 6 & 2,2 \\
Carcinoma Indiferenciado & 1 & 0,4 \\
\hline Total & 271 & 100,0 \\
\hline
\end{tabular}

publicada por un servicio de ORL en Chile. Es una cirugía segura con baja frecuencia de complicaciones.

Si bien, en nuestro centro se realizaron tiroidectomías parciales, éstas se realizaron en el contexto de patologías oncológicas de cabeza y cuello, cuya técnica quirúrgica incluye la resección de un lóbulo tiroideo, como es el caso del cáncer de laringe.

En el caso de patología tiroidea, en nuestro centro siempre se realiza tiroidectomía total, ya que no se ha demostrado que presente mayor tasa de complicaciones al compararla con una hemitiroidectomía, además que disminuye la frecuencia de recidivas, por lo que se realiza tiroidectomía total tanto para patología benigna como maligna.

Otra vía de abordaje, es la tiroidectomía video asistida. Sin embargo, no ha demostrado una diferencia estadísticamente significativa en relación a la prevención de hipocalcemia, lesión de NLR y hemorragia profusa si la comparamos con la tiroidectomía total clásica, pero sí ha demostrado una diferencia estadísticamente significativa en relación al resultado estético y al dolor posoperatorio. Otro punto en contra, es que con esta técnica no se puede realizar una disección cervical del compartimento central de forma adecuada. Por lo que, dentro de las contraindicaciones de este abordaje, es la presencia de nódulos mayores de 3 centímetros y adenopatías en compartimiento central ${ }^{10}$.

En este reporte ampliado de casos de tiroidectomías totales, se mantiene lo publicado por Ortega y cols ${ }^{1}$ en 2011 con respecto a realizar la sección de los músculos pretiroideos sin aumentar la morbilidad, y así lograr una mejor exposición quirúrgica. A su vez, el uso de la Harmonic Scalpe Focus $^{\circledR}$, ha disminuido el tiempo quirúrgico, sin evidenciar una mayor tasa de complicaciones ${ }^{8}$. El uso de drenaje aspirativo subcutáneo, sigue siendo una práctica habitual por el equipo quirúrgico, con resultados satisfactorios.

Otro factor que ha influido en disminuir el tiempo quirúrgico ha sido la curva de aprendizaje y la cohesión del equipo, logrando disminuir en promedio 20 minutos la duración de la cirugía.

Las 2 complicaciones más frecuentes de la tiroidectomía total son la hipocalcemia y la lesión del NLR, las cuales se pueden presentar hasta en el $40 \%$ y $7 \%$, respectivamente. En nuestra serie de casos la tasa de complicaciones se encuentra dentro de los rangos publicados en la literatura, siendo de $25 \%$ y de $3 \%$, respectivamente.

El tamaño del nódulo tiroideo y el diagnóstico histológico no influyeron en el desarrollo de complicaciones $(p>0,05)$. Las complicaciones se presentan con mayor frecuencia en los pacientes en los que se realiza disección ganglionar del nivel $\mathrm{VI}^{11}$. En nuestra serie el $25,1 \%$ de TT se complicaron vs $54,8 \%$ de las TT asociadas a disección ganglionar ( $\mathrm{p} 0,002)$.

El aporte posoperatorio de calcio oral de forma sistemática nos ha ayudado en prevenir el desarrollo de hipocalcemias severas y sintomáticas ${ }^{9}$. Los pacientes a los que se les realizó vaciamiento cervical y en aquellos en que se observaron 20 menos paratiroides, presentaron mayor tasa de hipocalcemia. 


\section{CONCLUSIÓN}

La tiroidectomía total es una técnica segura cuando la realiza un equipo con experiencia. Es de elección tanto en patología maligna como benigna,

\section{BIBLIOGRAFÍA}

1. Ortega P, Urra A, Compán A. Experiencia clínica en tiroidectomía total. Rev Otorrinolaringol Cir Cabeza Cuello 2011; 71: 53-6.

2. Rogers-Stevane J, Kauffman GL Jr. A historical perspective on surgery of the thyroid and parathyroid glands. Otolaryngol Clin North Am 2008; 41: 1059.

3. Cohen J, Clayman G. Atlas of head and neck surgery. Chapter 47. Subtotal and Total Thyroidectomy. Pag 465-474. Edit. Elsevier 2014.

4. Asari R, Passler C, et al. Hypoparathyridism after total thyroidectomy. A prospective study. Arch Surg 2008; 143 (2): 132-7.

5. Shindo M, ChHeda N. Incidence of vocal cord paralysis with and with out recurrent laryngeal nerve monitoring during thyroidectomy. Arch Otolaryngol Head neck Surg 2007; 133: 481-5.

6. Pérez J, Urrutia V, y cols. Uso selectivo de drenajes en tiroidectomías. Rev Chilena de Cirugía 2009; 61(6): 515-8. presentando una baja tasa de complicaciones. Nuestra experiencia presenta resultados concordantes con los estándares aceptados por la literatura mundial en relación a indicaciones, diagnósticos y complicaciones.

7. Tuttle M. Differentiated thyroid cancer: Surgical Treatment. UpToDate June 2015. Revisado el 16 de julio de 2015. Disponible en: www.uptodate.com.

8. Ecker T, Lopes Carvalho A, Jun-Ho C, et al. Hemostasis in Thyroid Surgery: Harmonic Scalpel vs other techniques - a metanalysis. Otolaryngology Head and Neck Surgery 2010; 143: 17-25.

9. RoH J, PARK J, PARK C. Prevention of postoperative hipocalcemia with rutine oral calcium and vitamin D supplements in patients with differentiatied papillary thyroid carcinoma undergoing total thyroidectomy plus central neck dissection. Cancer 2009; 115: 251-8.

10. Cohen J, CLAYMan G. Atlas of head and neck surgery. Chapter 48. Video-Assisted Thyroidectomy. Pag 475-487. Edit. Elsevier 2014.

11. Shindo M, Stern A. Total thyroidectomy with and without selective central compartament dissection. A comparision of complication rates. Arch Otola-ryngol Head Neck Surg 2010; 136(6): 584-7.

Dirección: Gabriel Undurraga Perl. 